\title{
Are passive red spirals truly passive? (Research Note)
}

\section{The current star formation activity of optically red disc galaxies}

\author{
L. Cortese
}

\begin{abstract}
European Southern Observatory, Karl-Schwarzschild-Str. 2, 85748 Garching bei München, Germany e-mail: 1cortese@eso.org
\end{abstract}

Received 19 April 2012 / Accepted 30 May 2012

ABSTRACT

\begin{abstract}
We used GALEX ultraviolet and WISE $22 \mu \mathrm{m}$ observations to investigate the current star formation activity of the optically red spirals recently identified as part of the Galaxy Zoo project. These galaxies were accurately selected from the Sloan Digital Sky Survey as pure discs with low or no current star formation activity, representing one of the best optically selected samples of candidate passive spirals. However, we show that these galaxies are not only still forming stars at a significant rate $\left(\gtrsim 1 M_{\odot} \mathrm{yr}^{-1}\right)$ but, more importantly, their star formation activity is not different from that of normal star-forming discs of the same stellar mass $\left(M_{*} \gtrsim 10^{10.2} M_{\odot}\right)$. Indeed, these systems lie on the UV-optical blue sequence, even without any corrections for internal dust attenuation, and they follow the same specific star formation rate vs. stellar mass relation of star-forming galaxies. Our findings clearly show that at high stellar masses, optical colours do not allow to distinguish between actively star-forming and truly quiescent systems.
\end{abstract}

Key words. galaxies: evolution - galaxies: photometry - galaxies: star formation - ultraviolet: galaxies - infrared: galaxies

\section{Introduction}

Since its discovery, the colour vs. magnitude (or stellar mass) relation (e.g., Visvanathan \& Sandage 1977; Visvanathan 1981) has been adopted as one of the most useful tools for characterizing the properties of galaxies across the Hubble time. Particularly powerful is the use of colours to distinguish between late-type/star-forming systems and early-type/passive galaxies, when accurate morphological classification is not available. However, it is now well known that the bimodality in the colour distribution of galaxies does not always reflect a difference in morphological type (Scodeggio et al. 2002; Franzetti et al. 2007). Indeed, at least in the local Universe, blue/starforming spheroids (e.g., Yi et al. 2005; Kannappan et al. 2009; Schawinski et al. 2009) and red/quiescent spirals (e.g., van den Bergh 1976; Moran et al. 2006; Crowl \& Kenney 2008; Cortese \& Hughes 2009; Wolf et al. 2009; Bundy et al. 2010; Rowlands et al. 2012) do exist.

In the last decade, we have seen a rapid increase in the number of studies focused on these unusual systems with the aim of understanding why they do not follow the typical relations between morphology and colours. However, while identifying current star formation activity in elliptical galaxies is relatively easy, isolating truly quiescent disc galaxies is a more complicated problem. Indeed, optical red colours do not always automatically imply a passive stellar population (e.g., Cowie \& Barger 2008; Cortese \& Hughes 2009). Dust extinction and a large bulge component are among some of the problems that can significantly affect the observed optical colours of discs, making them appear much more "evolved" than they really are. For these reasons, the vast majority of the works focused on truly passive spirals have taken advantage of multiwavelength datasets spanning from the ultraviolet to the mid- and far-infrared regime to account at least for the effects of internal dust absorption (e.g., Wolf et al. 2009; Gallazzi et al. 2009).

Recently, as part of the Galaxy Zoo project (Lintott et al. 2008), Masters et al. (2010, hereafer M10) performed a careful selection to isolate and study red spirals. These authors took advantage of the morphological classification available for thousands of objects to select spiral galaxies and then adopted a simple cut in inclination, optical colour, and bulge fraction to identify disc-dominated objects that should be "truly passive" (i.e. with current star formation activity significantly lower than what is observed in normal spiral galaxies of similar stellar mass). The novelty of this technique lies in the fact that only optical data, combined with an accurate morphological classification, are needed to isolate the red spiral population. If successful, these selection criteria may significantly facilitate isolating passive systems, thus improving our understanding of the evolutionary paths leading to these unusual galaxies.

Thus, in this Research Note we take advantage of GALaxy Evolution eXplorer (GALEX, Martin et al. 2005) ultraviolet (UV) and Wide-field Infrared Survey Explorer (WISE, Wright et al. 2010) $22 \mu \mathrm{m}$ observations to study the star formation properties of these optically red spiral galaxies and to determine whether they really are passive.

\section{The data}

The sample of optically red spirals presented by M10 comes from the Galaxy Zoo clean catalogue (Lintott et al. 2008), which is based on the Sloan Digital Sky Survey (SDSS) DR6 (Adelman-McCarthy et al. 2008). First, a volume-limited sample of spirals was obtained assuming spiral likelihood ${ }^{1}$

1 This corresponds to the fractional number of cases in which a galaxy has been classified as a spiral by the public. 
(Bamford et al. 2009) greater than 0.8, spectroscopic redshift $0.03<z<0.085$ and $r$-band absolute magnitude $M_{r}<$ -20.17 mag. Secondly, bulge-less and face-on discs were selected requiring a ratio between the major and minor axis of the galaxy $\log (a / b)<0.2$ and a fraction of the light coming from the de Vaucouleur component of the surface brightness profile fracDev $<0.5$. Finally, red spirals were defined as objects with a colour $(g-r)>0.63-0.02 \times\left(M_{r}+20\right)$. The sample of red spirals so obtained includes 294 galaxies.

Unfortunately, not all galaxies in the M10 sample are included in the GALEX GR6 public release. Thus, in the following analysis, we only focus on the subset of 255 galaxies observed by GALEX and lying within $0.55^{\circ}$ from the centre of a GALEX tile to avoid edge effects ${ }^{2}$. Given that this subset includes $\sim 87 \%$ of the original sample, we are confident that our conclusions can be extended to the whole population of optically red galaxies studied by M10.

\subsection{GALEX and WISE photometry}

We cross-correlated the 255 red spirals with the WISE all-sky survey source catalogue using a matching radius of 10 arcsec. We found 233 galaxies detected at $22 \mu \mathrm{m}$ but, after a careful visual inspection of the images, 46 turned out to be spurious or marginal detections, or in regions highly contaminated by foreground emission. Thus, out of the 255 galaxies in our sample, $166(\sim 65 \%)$ are clearly detected at $22 \mu \mathrm{m}$.

To obtain homogeneous flux density estimates in the GALEX UV and WISE $22 \mu \mathrm{m}$ bands, we performed aperture photometry using the same aperture size in all images. We adopted circular apertures of size twice the isophotal diameter at the 25 mag $\operatorname{arcsec}^{-2}$ level in $r$ band, convolved to the WISE $22 \mu \mathrm{m}$ resolution (12 arcsec). In a few cases, the size of the aperture has been adjusted to make sure that the entire emission from the galaxy is included. Sky background was determined in circular annuli or boxes around the target, and foreground/background objects were accurately masked.

All 255 galaxies in our sample are detected in nearultraviolet (NUV), while only for 220 objects $(86 \%$ of the sample) we were able to obtain a far-ultraviolet (FUV) flux density. This is mainly because the FUV observations are in general less sensitive than the NUV ones. By comparing the magnitudes obtained from independent observations of the same galaxy, as well as with the GALEX GR6 public catalogue, we obtain a typical uncertainty of $\sim 0.15$ and $0.30 \mathrm{mag}$ in NUV and FUV, respectively. Similarly, we find a very good agreement between our flux densities and the standard aperture estimates (w4MAG) in the WISE all-sky survey catalogue, with a typical scatter of $\sim 0.2 \mathrm{mag}$.

UV magnitudes have been corrected for Galactic extinction following Wyder et al. (2007). No aperture or colour corrections were applied to the $22 \mu \mathrm{m}$ flux densities. These are in general lower than the uncertainty on the flux density, and thus do not affect our results.

\section{Results}

Figure 1 shows where the red spirals (red filled circles) lie in an $N U V-r$ colour vs. stellar mass $\left(M_{*}\right)$ diagram. Stellar masses are taken from the MPA/JHU SDSS DR7 release ${ }^{3}$. These are derived from SDSS photometry using the spectral energy

\footnotetext{
${ }^{2}$ Only seven galaxies in the sample have been observed but lie at distances greater than $0.55^{\circ}$.

${ }^{3}$ http://www .mpa-garching .mpg.de/SDSS/DR7/
}

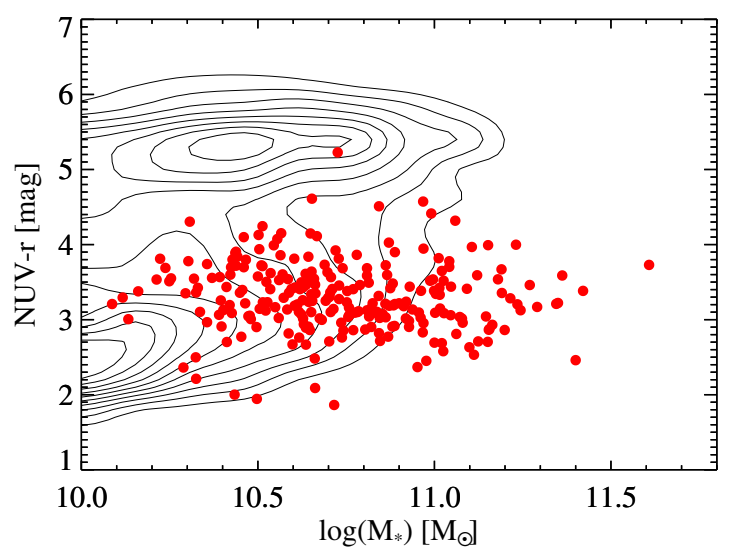

Fig. 1. Optically red spirals (red circles) on a $N U V-r$ vs. stellar mass diagram. The contours show the distribution of galaxies in the GASS survey (Catinella et al. 2010).

distribution fitting technique described in Salim et al. (2007), assuming a Chabrier (2003) initial mass function (IMF). The black contours show the colour distribution of the parent sample of the GALEX Arecibo SDSS survey (GASS, Catinella et al. 2010), a volume-limited sample of nearby massive galaxies $\left(M_{*}>10^{10} M_{\odot}\right)$, providing an indication of the typical range of UV colours of red- and blue-sequence massive galaxies in the local Universe.

Clearly, the red spirals in M10 sample are not quiescent. Not only is there just one galaxy in the UV red sequence, but almost the whole sample ( $94 \%$, i.e., 240 out of the 255 galaxies) has an observed $N U V-r$ colour bluer than $4 \mathrm{mag}$, i.e., the typical value observed in actively star-forming galaxies (e.g., Salim et al. 2007).

To determine if the current star formation activity of these optically red spirals is different from that of normal starforming galaxies of similar stellar mass, we determined star formation rates (SFRs) by combining the GALEX UV and WISE $22 \mu \mathrm{m}$ data. We used the $22 \mu \mathrm{m}$ fluxes to correct the NUV and FUV luminosities for dust attenuation following Hao et al. (2011):

$$
\begin{aligned}
L(\mathrm{NUV})_{\mathrm{corr}} & =L(\mathrm{NUV})+2.26 \times L(22 \mu \mathrm{m}) \\
L(\mathrm{FUV})_{\mathrm{corr}} & =L(\mathrm{FUV})+3.89 \times L(22 \mu \mathrm{m}),
\end{aligned}
$$

where $L$ are luminosities in units of $\mathrm{erg} \mathrm{s}^{-1}$. It is important to note that these relations were calibrated using Spitzer $24 \mu \mathrm{m}$ and IRAS $25 \mu \mathrm{m}$ flux densities, whereas in this case we are applying them to the WISE $22 \mu \mathrm{m}$ emission. Because for typical starforming objects the $22 \mu \mathrm{m}$ emission should be comparable to, or slightly fainter than, the $24 \mu \mathrm{m}$ flux density, we can assume our corrections to be a lower limit to the real value. For galaxies observed but not detected by WISE we considered two different extreme cases by either fixing the $22 \mu \mathrm{m}$ emission to zero or to the typical $3 \sigma$ sensitivity limit of WISE (i.e. $3 \mathrm{mJy}$ ). We then estimated the current SFR from the corrected UV luminosities using the relation presented by Salim et al. (2007) that was calibrated on a Chabrier (2003) IMF

$\operatorname{SFR}\left(M_{\odot} \mathrm{yr}^{-1}\right)=1.46 \times 10^{-28} \times L_{v}$

where $L_{v}$ is in units of $\mathrm{erg} \mathrm{s}^{-1} \mathrm{~Hz}^{-1}$. The SFRs estimated from the NUV and FUV luminosities agree within $\sim 10-25 \%$, with the NUV-based SFR being typically higher by $\sim 0.03-0.07$ dex.

In Fig. 2 we plot the specific star formation rate $(S S F R=$ $S F R / M_{*}$ ) as a function of the stellar mass for the M10 sample. 


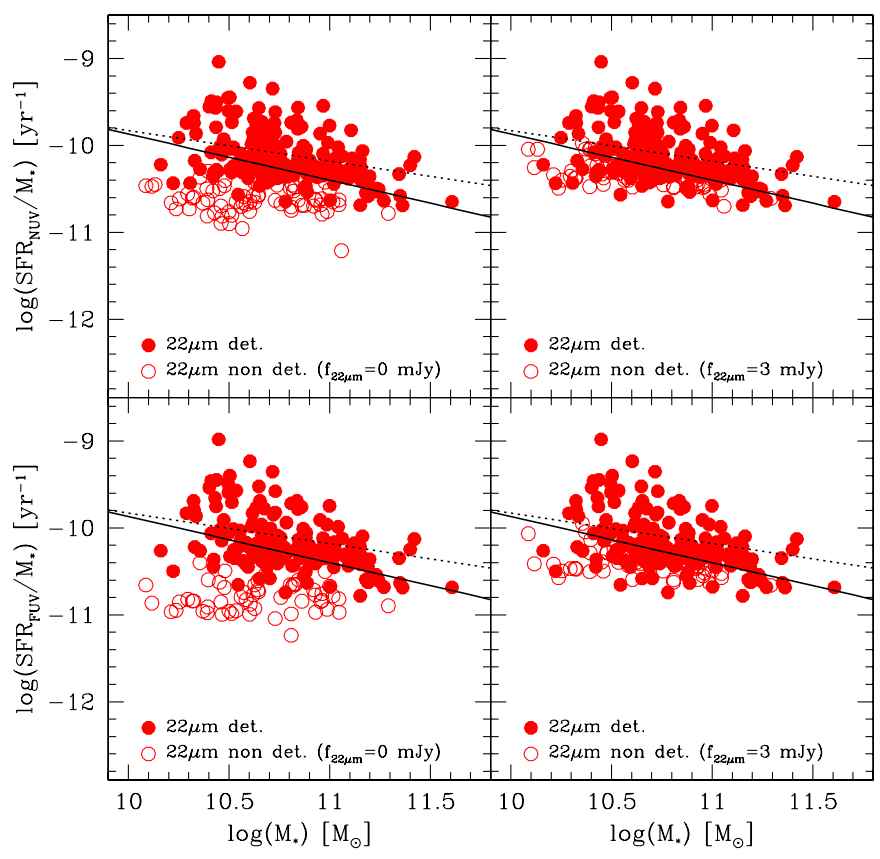

Fig. 2. NUV- (top) and FUV-based (bottom) specific star formation rate vs. stellar mass relation for the sample of optically red spirals. Filled and empty circles show $22 \mu \mathrm{m}$ detections and non-detections, respectively. For non-detections, the SFR has been determined by assuming a $22 \mu \mathrm{m}$ flux density equal to zero (left panels) or to $3 \mathrm{mJy}$ (i.e., $\sim 3 \sigma$ WISE detection limit; right panels). The dotted and solid lines show the best fit to the star-forming sequence obtained by Salim et al. (2007) by including only star-forming galaxies (i.e., excluding galaxies with optical lines ratios typical of active galactic nuclei) or all galaxies with $N U V-r$ colour lower than 4 , respectively.

The top and bottom panels show the results obtained for the NUV-based and FUV-based star formation rates, respectively. For each row, the difference between the left and right panels is in the treatment of the WISE non detections. While in the left panels the $22 \mu \mathrm{m}$ flux density is set to zero, in the right ones it is equal to the typical $3 \sigma$ noise level of WISE images. Thus, the two panels show the two extremes, with the real relation followed by this sample lying in between the two plots.

As expected from what we already showed in Fig. 1, and regardless of the way we treat non-detections, all red spirals are forming stars at a significant rate and $\sim 85-90 \%$ (depending on which SFR estimate is used) have a current $S F R \geq 1 M_{\odot} \mathrm{yr}^{-1}$. More importantly, the M10 sample follows the relation obtained for nearby star-forming galaxies by Salim et al. (2007, solid and dashed lines in Fig. 2) remarkably well, implying that these galaxies are not different from the local population of blue sequence discs.

\section{Discussion and conclusion}

The results presented in the previous section clearly show that the red spirals in the M10 sample are not passive but are still forming stars at the rate expected for their stellar mass. It is thus important to briefly discuss why the very careful optical selection performed by M10 did not isolate truly quiescent systems.

We can safely exclude that dust attenuation plays a dominant role. The selection of face-on spirals guarantees the absence of highly obscured systems, as clearly shown in Fig. 1. Indeed, dust extinction would much more heavily affect the UV colours and move all galaxies to the UV red sequence, contrary to what is observed.
To understand why these objects have optically red colours despite their still on-going star formation activity, it is important to remember that they are massive galaxies. For stellar masses $\gtrsim 10^{10} M_{\odot}$, the optical blue cloud merges into the red sequence, suggesting that optical colours are no longer a good proxy for the current star formation activity of a galaxy. Indeed, as extensively discussed by Wyder et al. (2007), in the UV the blue and red sequences are well separated even at high luminosities, implying that massive spirals are forming stars despite what is suggested by their optical colours. This is, at least partially, a consequence of the fact that massive objects formed the bulk of their stars at earlier epochs than dwarf galaxies (e.g., Boselli et al. 2001; Gavazzi et al. 2002; Heavens et al. 2004; Thomas et al. 2010), and that optical colours are more directly related to the average age of the stellar populations than to the current star formation activity (e.g., Wyder et al. 2007; Chilingarian $\&$ Zolotukhin 2012). The fact that the fraction of the optically red spirals in the M10 sample significantly increases with stellar mass (see Fig. 2 of M10) is consistent with this scenario.

In conclusion, at least at high stellar masses, optical colours alone are not sufficient to distinguish between actively starforming and quiescent systems.

Acknowledgements. I wish to thank Daniel Thomas for encouraging me to publish this study and for useful comments, and Barbara Catinella for providing the data from the GASS survey. GALEX (Galaxy Evolution Explorer) is a NASA Small Explorer, launched in April 2003. We gratefully acknowledge NASA's support for construction, operation, and science analysis for the GALEX mission, developed in cooperation with the Centre National d'Études Spatiales (CNES) of France and the Korean Ministry of Science and Technology. This publication makes use of data products from the Wide-field Infrared Survey Explorer, which is a joint project of the University of California, Los Angeles, and the Jet Propulsion Laboratory/California Institute of Technology, funded by the National Aeronautics and Space Administration. The research leading to these results has received funding from the European Community's Seventh Framework Programme (/FP7/2007-2013/) under grant agreement No. 229517.

\section{References}

Adelman-McCarthy, J. K., Agüeros, M. A., Allam, S. S., et al. 2008, ApJS, 175, 297

Bamford, S. P., Nichol, R. C., Baldry, I. K., et al. 2009, MNRAS, 393, 1324

Boselli, A., Gavazzi, G., Donas, J., \& Scodeggio, M. 2001, AJ, 121, 753

Bundy, K., Scarlata, C., Carollo, C. M., et al. 2010, ApJ, 719, 1969

Catinella, B., Schiminovich, D., Kauffmann, G., et al. 2010, MNRAS, 403, 683

Chabrier, G. 2003, PASP, 115, 763

Chilingarian, I. V., \& Zolotukhin, I. Y. 2012, MNRAS, 419, 1727

Cortese, L., \& Hughes, T. M. 2009, MNRAS, 400, 1225

Cowie, L. L., \& Barger, A. J. 2008, ApJ, 686, 72

Crowl, H. H., \& Kenney, J. D. P. 2008, AJ, 136, 1623

Franzetti, P., Scodeggio, M., Garilli, B., et al. 2007, A\&A, 465, 711

Gallazzi, A., Bell, E. F., Wolf, C., et al. 2009, ApJ, 690, 1883

Gavazzi, G., Bonfanti, C., Sanvito, G., Boselli, A., \& Scodeggio, M. 2002, ApJ, 576,135

Hao, C.-N., Kennicutt, R. C., Johnson, B. D., et al. 2011, ApJ, 741, 124

Heavens, A., Panter, B., Jimenez, R., \& Dunlop, J. 2004, Nature, 428, 625

Kannappan, S. J., Guie, J. M., \& Baker, A. J. 2009, AJ, 138, 579

Lintott, C. J., Schawinski, K., Slosar, A., et al. 2008, MNRAS, 389, 1179

Martin, D. C., Fanson, J., Schiminovich, D., et al. 2005, ApJ, 619, L1

Masters, K. L., Mosleh, M., Romer, A. K., et al. 2010, MNRAS, 405, 783

Moran, S. M., Ellis, R. S., Treu, T., et al. 2006, ApJ, 641, L97

Rowlands, K., Dunne, L., Maddox, S., et al. 2012, MNRAS, 419, 2545

Salim, S., Rich, R. M., Charlot, S., et al. 2007, ApJS, 173, 267

Schawinski, K., Lintott, C., Thomas, D., et al. 2009, MNRAS, 396, 818

Scodeggio, M., Gavazzi, G., Franzetti, P., et al. 2002, A\&A, 384, 812

Thomas, D., Maraston, C., Schawinski, K., Sarzi, M., \& Silk, J. 2010, MNRAS 404, 1775

van den Bergh, S. 1976, ApJ, 206, 883

Visvanathan, N. 1981, A\&A, 100, L20

Visvanathan, N., \& Sandage, A. 1977, ApJ, 216, 214

Wolf, C., Aragón-Salamanca, A., Balogh, M., et al. 2009, MNRAS, 393, 1302

Wright, E. L., Eisenhardt, P. R. M., Mainzer, A. K., et al. 2010, AJ, 140, 1868

Wyder, T. K., Martin, D. C., Schiminovich, D., et al. 2007, ApJS, 173, 293

Yi, S. K., Yoon, S.-J., Kaviraj, S., et al. 2005, ApJ, 619, L111 